\title{
FRIEDRICH SPEE E LA PRESUNZIONE D'INNOCENZA
}

\author{
Nota del m.e. MARIO PISANI (*)
}

(Adunanza del 4 ottobre 2018)

SunTO. - Nella sua celebre Cautio criminalis, per la prima volta edita nel 1631, il gesuita padre Friedrich Spee appare, un secolo e mezzo prima della Rivoluzione francese, come precursore del concetto di presunzione di innocenza. L'autore ne tratta sotto due profili: con riferimento alla vittima dei processi fondati su prove illecite e alla c.d. elisione degli indizi da valutarsi ai fini dell'applicazione della tortura. Per l'abolizione di quest'ultima il padre Spee si segnalerà come una delle figure più significative.

$* * *$

ABstRACT. - In his famous Cautio Criminalis, published for the first time in 1631, Jesuit Father Fiedrich Spee seems to have anticipated, a century and a half before the French Revolution, the idea of Presumption of Innocence. The Author deals with it below two profiles: with reference to the victim of trials based on illicit evidences and to the so called elision of the clues to be assessed for the application of torture. Father Spee will be reported as one of the most significant figures for the abolition of the torture.

1. La figura del padre gesuita tedesco Friedrich Spee von Langelfeld, nato nel 1591, era già stata presentata in questa sede, nell'adunanza 16 novembre del lontano 1972. Come risulta dai Rendiconti Letterari dell'Istituto (fasc. III del 1972, pp. 844-905) si trattava di un'ampia nota di Emmy Rosenfeld, introdotta da Vittore Pisani.

La nota faceva particolare riferimento, già nel titolo - Il «Theologischer Process» di Friedrich Spee von Langenfeld - ad un'opera

(") Professore emerito nella Facoltà di Giurisprudenza dell’Università degli Studi di Milano, Italy. E-mail: mariopisani.mp@libero.it 
della quale veniva dimostrata come corretta la già discussa attribuzione come autore all'indicato padre gesuita. Di quell'opera - che era stata pubblicata anonima, nel 1631, a Rinteln in Westfalia, nel formato di un volumetto in $8^{\circ}$ di due sedicesimi - in certo modo a titolo di eccezione nei nostri Rendiconti veniva pubblicato (pp. 850-880) l'originario testo integrale in lingua tedesca.

Il saggio che, a guisa di appendice vi faceva seguito (in lingua italiana), così illustrava la particolare finalità del testo: come il lettore può rendersi conto, "il «Processo teologico» è uno speculum spirituale a conforto di quei sacerdoti a cui era affidata la cura d'anime delle infelici «streghe» prigioniere, col compito di accompagnare le condannate nell'ultimo tragitto al rogo".

Più in particolare esso - si diceva - aveva "un compito limitato, ma importante: offrire consigli pratici e suggerire preghiere adatte a quei sacerdoti che svolgevano la loro cura d'anime presso i così detti «incolpati convinti e confessi»: in un ambiente cioè dove era considerato criminale mettere in dubbio l'esistenza della stregoneria".

2. Data la stretta connessione tematica, e per di più la contemporaneità della stampa di una prima edizione, il saggio d'appendice di cui s'è detto, a firma Rosenfeld, non mancava di offrire ampie indicazioni esplicative in ordine ad un altro, più ampio ed impegnativo, scritto del padre Spee, come già il Theologischer Process per la prima volta pubblicato anonimo, nel 1631, presso lo stesso editore Peter Lucius di Rinteln.

Si trattava dell'ormai celebre volume - e su questo vogliamo ora intrattenerci - intitolato Cautio Criminalis seu de Processibus contra sagas liber, al quale, a distanza di qualche secolo, resta affidata, dopo la riscoperta della sua paternità operata da Leibniz, la celebrità dell'autore (noto anche come "grande poeta di un canzoniere mistico" e di "meditazioni illuminate").

Se il testo indicato in precedenza aveva per destinatari i sacerdoti, quest'altro - come risulta anche dal sottotitolo - in principalità vedeva invece come destinatari i magistrati, e, in sequenza, i consiglieri dei prìncipi e i protagonisti e comprimari dei processi di stregoneria.

Servendosi, questa volta, "di un brillante latino classico" il padre Spee, già professore di filosofia nel collegio gesuita di Paderborn, e poi, nel 1627, supplente, al Tricoronatum di Colonia, nella classe di metafisica, qualche tempo dopo si accingeva appunto alla stesura della Cautio 
Criminalis nella quale egli si rivelerà come "feroce e audace oppositore" dei processi dei quali s'è detto. ${ }^{1}$

3. In un incontro, come il presente (e del resto come di consueto) a tempo necessariamente limitato, non è certo possibile dedicare le nostre attenzioni, sia pure in linea di sintesi, a tutto l'arco delle tematiche affrontate nel volume del quale stiamo parlando.

E piuttosto è parso opportuno limitarci, dopo una breve esposizione di carattere generale, ad un tema del tutto particolare e più specifico, che vede il gesuita padre Spee - è il caso di anticiparlo - come un suo noncelebrato precursore a livello mondiale: si tratta del tema della presunzione di innocenza nella struttura e nella dottrina del processo penale.

Ci facilita il compito una prima edizione dell'opera in lingua italiana, che, datata 1986, verrà più tardi riproposta nel $2004 .^{2}$

L'autore si presenta con un efficace proemio dall'andatura singolarmente paradossale: "Questo libro è dedicato alle autorità tedesche. Non a chi lo leggerà, bensì a chi non lo leggerà mai. Infatti, coloro che si ritengono in dovere di leggere quello che ho scritto qui sulla stregoneria, sono già in possesso di quanto il libro dovrebbe fornire loro: la capacità di essere accurati e diligenti nell'esaminare a fondo queste cause. I più trascurati, invece, quelli che non leggeranno mai questo libro, sono proprio coloro che dovrebbero leggerlo accuratamente".

1 FIORELLI, La tortura giudiziaria nel diritto comune, vol. II, Milano 1954, p. 231, nota (45) riferisce circa la pluralità delle edizioni dell'opera che hanno fatto seguito alla prima, delle quali non meno di sette in latino tra il 1631 e il 1731, e numerose altre in varie lingue europee. Altre edizioni, non certo escluse quelle in lingua tedesca, seguiranno fino ai nostri giorni. Per altre indicazioni, riguardanti anche il significato storico e il sèguito della Cautio criminalis nella dottrina tedesca v. PETERS, Strafprozess - Ein Lebrbuch, Karlsruhe 1952, p. 54. In particolare M. CATTANEO, Illuminismo e diritto penale, Milano 1993, p. 63, richiama l'influenza di quell'opera sul De Crimine Magiae, del 1701, di CHR. THOMASIUS. Dello stesso v. Friedrich von Spee e la filosofia del processo penale, in Arch. st. e giur. sardo di Sassari, 1994, p. 129.

2 A questa faremo qui costante riferimento, nella traduzione curata da Mietta Timi, che l'editrice Salerno ha presentato (Roma 2004) con introduzione e a cura di ANNA FOA, sotto il titolo I processi contro le streghe (Cautio criminalis), pp. 377. A commento della pubblicazione v. MAGRIS, L'avvocato delle streghe, in Corriere della Sera del 16 novembre 2014, p. 30 , a giudizio del quale - v. nello stesso quotidiano, in data 31 maggio 2018 - Spee "dovrebbe essere considerato uno dei grandi dell'umanità".

3 Viene ricordato il caso - v. VENTURI, Settecento riformatore - Da Muratori 
Il padre Spee affronta il complesso e difficile argomento con modalità dialettiche, nella forma di questioni - in totale nel numero di cinquantuno - comprensive di un'ampia e corposa serie di corollari. Le questioni si sviluppano secondo l'ordine logico delle successive approssimazioni. ${ }^{4}$

L'autore comincia col chiedersi (questione I) se "esistono davvero uomini e donne che praticano la stregoneria", e risponde che se, a suo giudizio, "sulla terra esistono effettivamente alcune persone che praticano la stregoneria", non può però credere che fossero "tutte streghe quelle che finora sono finite in cenere". ${ }^{5}$ Passa poi ad esprimere una risposta positiva, ma in termini di probabilità, alla questione, per noi minore, intitolata: "Le streghe e i maghi sono davvero più numerosi in Germania che altrove?"

$\mathrm{Al}$ più importante quesito - questione III - "Di che natura sia il delitto di stregoneria", il padre Spee brevemente risponde trattarsi "di un delitto enorme, gravissimo, terribile”, ma fa però salva la necessità di "una analisi nuova e accurata".

Viene quindi configurata una risposta d'ordine affermativo, in

a Beccaria, Torino 1969, p. 371 - di una giovane di anni sedici, allieva di una lavandaia già decapitata in Baviera, e sottoposta a Salisburgo, col rischio della vita, a un processo per stregoneria(1751). Si menzionava l'iniziativa (... rimasta però senza successo) di una persona "addottrinata e capace" che s'era adoperata "validamente" a favore della giovane, anche facendo "leggere da' giudici il padre Spee...".

4 Già per queste ragioni risulta alquanto riduttiva la presentazione dell'opera accennata da CORDERO, Guida alla procedura penale, Torino 1986, p. 198, semplicemente a guisa di un "impolitico cri de coeur sugli abusi antistregoneschi". E cioè quasi dunque si trattasse di un saggio di tipo umanitario, che - a dire dello stesso critico (Criminalia - Nascita dei sistemi penali, Bari 1985, p. 339 ss.) - avrebbe goduto di una "fortuna immeritata". Cfr. PAPA, "A chi legge": l'incipit visionario Dei delitti e delle pene, in Criminalia, 2014, p. 641, il quale parla di "acuta e appassionata critica contro il livore oscurantista della caccia alle streghe e contro l'uso della tortura; pagine che anticipano di oltre un secolo gli argomenti di Beccaria". V. anche FIORELLI, op. cit., p. 232, dove si parla di libro "originalissimo e potente ... La Cautio criminalis non nega fede alla possibilità della stregoneria, in astratto, ma la nega nel modo più formale a ciò che della stregoneria si legge nei più celebrati scrittori della materia". Ricordiamo che nella lettera ai Gálati $(5,20)$ Paolo annoverava "le stregonerie" entro il cospicuo numero delle "opere della carne".

5 Ed aggiungeva: “... E come me, non lo credono molti uomini timorati e giusti", auspicando nel lettore un "atteggiamento razionale ... in nome di quella carità che il nostro legislatore Gesù Cristo desiderava ardentemente che si accendesse nel cuore dei suoi fedeli”. 
conformità con gli "studiosi del diritto", alla questione IV - "Se la stregoneria possa considerarsi un delitto eccezionale" - , nel senso che essa va annoverata tra i reati "particolarmente pericolosi - si noti - per lo Stato, contro i quali sembra opportuno intervenire con misure eccezionali". 6

Il discorso del padre Spee diventa più serrato nel dare poi risposta alla questione $\mathrm{V}$, ovvero al quesito "Se sia legittimo istruire una procedura arbitraria in presenza di delitti eccezionali" . Questo l'esordio: "La mia opinione è che non sia legittimo".

Seguono gli sviluppi argomentativi, orientati verso la configurazione di un "principio generale "a guisa di "un assioma inderogabile", del seguente tenore: se i delitti sono "eccezionali rispetto al diritto positivo (...) non lo sono comunque rispetto alla ragione e alla legge naturale. Quindi, qualsiasi processo si istruisca contro questi delitti, con procedura sia ordinaria sia straordinaria, si deve necessariamente svolgere in modo tale da non essere in contrasto con la ragione e con la morale".

Ed ancora: "Non è assolutamente vero, quindi, che sia lecito, nei delitti eccezionali, prescindere tranquillamente da ogni disposizione prevista dal diritto positivo. Si può derogare da alcune disposizioni, ma non da tutte".

A supporto di questa tesi l'autore richiama l'insegnamento di Prospero Farinacio, ovvero del celebre autore di Praxis et theoricae criminalis libri duo, editi a Venezia nel 1603, oltre che dei molti giuristi da lui ricordati.

Per ragioni di brevità proseguiamo il nostro percorso - omesse quelle intermedie - concentrandoci sull'undicesima tra le questioni che, in diretta prosecuzione della $\mathrm{X}$ (così formulata: " $E$ ' possibile che talvolta Dio consenta anche il coinvolgimento di innocenti?"), si sostanzia nella risposta al seguente ed importante quesito: " $E$ ' possibile che di fatto si sia permesso il coinvolgimento di persone innocenti?"

Il nostro autore introduce la sua risposta in modo lapidario: "Binsfeld e Del Rio pare non siano dello stesso parere". ${ }^{7}$ Ma chi sono costoro? E' allora il caso di rappresentare, sulla scorta di quanto precisato dalla curatrice della recente versione della Cautio già ricordata,

6 Facciamo riferimento alla versione della Cautio indicata nella nota (2), pp. 47 ss.

7 Dobbiamo far capo alle pp. 73 ss. del volume qui sopra indicato.

8 Si veda l'introduzione, a p. 28. 
che, dei due accreditati demonologi, il primo, Binsfeld, vescovo di Treviri, nel 1589 aveva pubblicato il Tractatus de confessionibus maleficorum et sagarum, mentre quanto al secondo, il Del Rio, giurista e poi gesuita, ${ }^{9}$ non minore successo riportò con la pubblicazione a Lovanio, la prima volta nel 1599-1600, di un suo volume intitolato Disquisitionum magicarum libri sex.

L'autore della Cautio comincia col rispondere: "Ma non v'è dubbio che, molto spesso, persone innocenti sono state coinvolte nella stessa sorte di quelle colpevoli”.

Segue, articolata in dieci punti, la motivazione di tale risposta.

A noi qui preme intrattenerci sul punto n. 9, che però, a sua volta, necessita di un preliminare chiarimento in merito alla c.d. prova dell'acqua, adottata temporibus illis, in Westfalia e nei territori renani. Questa la descrizione offerta proprio da Binsfeld: "La strega presunta è condotta ad un fiume o ad un lago, è legata mani e piedi ed è gettata nell'acqua fredda. Se galleggia, è fortemente sospetta di essere una strega - commento nostro: la stregoneria ... vince la forza di gravità - e questo è un indizio per ripetere la tortura. Se va a fondo viene assolta come innocente" .... ${ }^{10}$

Dalle "stesse parole di Binsfeld e Del Rio - puntualizza allora il padre Spee - si deduce che, di fatto, Dio ha permesso la morte di moltissime persone innocenti di questo delitto".

Segue la dimostrazione: i due stimatissimi demonologi sostengono, giustamente, che "la prova dell'acqua, per le streghe, è illecita, e se qualche giudice si basa su di essa per condurre l'istruttoria, questa è illecita e dunque il processo risulta nullo". Ma se così è - incalza il nostro autore - ne consegue che, "qualora si sia proceduto per questa via contro qualcuno, egli è morto innocente. Infatti - ed è questo il punto centrale della nostra riflessione -, una persona deve ritenersi innocente fintantochè non si sia legittimatamente provato che è colpevole”. Detto in altri termini: la prova illecita non è prova legittima, e l'itinerario di accertamento che su di essa si fonda è un processo non legale, un non-processo, e come tale inidoneo a superare la presunzione di partenza che sta a favore dell'imputato.

9 VENTURI, op. cit., p. 368, lo presenterà come "il più famoso e il più fantasioso persecutore di streghe tra i teologi del passato".

10 V. FOA, a p. 28 dell'introduzione cit. a nota (2). 
Perché nulla pena si può dare - diciamo noi oggi, integrando un vecchio e classico aforisma - sine legali judicio.

Il gesuita padre Spee, dunque, che pubblica nel 1631, se non proprio il padre nobile della presunzione d'innocenza (per giungere a tanto sarebbe necessaria qualche ricerca più approfondita), di quella nozione, di enorme importanza per la storia e per la struttura del processo penale nel volger dei secoli, attraverso sviluppi ed esiti discordi e discontinui, è almeno da ritenersi un suo precursore. Un precursore - si noti - anche rispetto agli immortali princìpi della Rivoluzione francese, vero essendo che dovrà passare ancora un secolo e mezzo prima che in proposito intervenga la Dichiarazione dei diritti dell'uomo e del cittadino, datata 26 agosto 1789.

$\mathrm{Va}$ anche notato che l'art. IX di questa Dichiarazione, dando soltanto per presupposta la regola di giudizio che discende da quella presunzione, pone più particolarmente l'accento sulla presunzione in discorso in quanto consequenziale regola di trattamento dell'imputato, specie per quanto concerne l'applicazione e l'esecuzione della carcerazione preventiva. Leggiamo: "Dovendosi presumere innocente ogni uomo sino a che non sia stato dichiarato colpevole - questa la premessa, come esplicito ma ancora grezzo presupposto fondativo, secondo quella dichiarazione, di una regola di giudizio - se il suo arresto sarà giudicato indispensabile deve però - questa la regola di trattamento che più in particolare sembra premere ai rivoluzionari - essere represso ogni rigore, che non sia necessario per assicurarsi della sua persona”.

4. Di contro alla presunzione di colpevolezza, che nettamente caratterizzava il processo di stampo inquisitorio, - diciamo noi oggi sul piano storico -, la presunzione di innocenza configura una regola base di giudizio, in quanto regola di verità: come a dire che, per affermare la colpevolezza è necessario poter superare, sul piano probatorio, la presunzione secondo cui l'imputato è da ritenersi, all'inizio e nel corso del processo, come presuntivamente non colpevole.

Da quella regola-base scaturisce, per una sorta di partenogenesi, una regola minore, quale criterio orientativo di scelta tra ipotesi di soluzioni alternative. Si tratta del criterio che tradizionalmente verrà poi designato favor rei, intendendosi come reus il soggetto de cuius agitur.

Ma per ritornare, più precisamente, al testo del precursore padre Spee, va detto che il medesimo, dopo aver prospettato, trattando la questione XVIII - "Quali corollari si possano trarre dalle ultime riflessioni” - , giunto all'enunciazione del XIV corollario affronta il tema 
della tortura giudiziaria, ovvero della tortura, a quei tempi legittima, se fondata su indizi legittimi. "Ammesso che - e il padre Spee, sia pure usando un linguaggio ancora un po' acerbo, non fa esclusivo riferimento ai sospettati di stregoneria - (ammesso che) un imputato sia passibile di tortura in base a legittimi indizi, non dovrà esservi sottoposto - egli scrive - in presenza di altri indizi contrari che provino la sua innocenza quanto i precedenti provavano la sua colpevolezza".

Era quella che possiamo definire come la "elisione degli indizi".

Si tratta di una presa di posizione, a supporto della quale il padre Spee mette in campo l'autorevolezza del Menochio (ovvero del suo De praesumptionibus, del 1609) e del Mascardi (ovvero delle sue Conclusiones omnium probationum, del 1593): una presa di posizione tanto più ragguardevole per il fatto che, come qualche secolo prima aveva scritto Bartolo da Sassoferrato ${ }^{11}$ - poi seguito da Claro - Doctores communiter dicunt quod in hoc non potest dari certa doctrina, sed relinquitur arbitrio judicis. ${ }^{12}$

Ma il padre Spee va oltre: di contro all'ancóra non certa dottrina, e richiamando l'autorità di Farinacio, e l'opinione dei dottori da lui citati "in gran numero", egli oppone che l'elisione, e neutralizzazione, degli indizi di cui s'è detto, "vale anche nel caso che gli indizi a favore dell'imputato siano leggermente inferiori a quelli sfavorevoli". Et alibi-scriveva appunto Farinacio, erroneamente e ingiustamente poi accusato, nel famoso incipit di "Dei delitti e delle pene", addirittura come propenso a suggerire tormenti "con iraconda compiacenza" 13 - saepe Doctores, qui communiter in proposito concludunt, quod si indicia pro reo existentia sint graviora, vel paria, vel etiam aliquantulum leviora, E debiliora indiciis contra reum existentibus, non potest ullo pacto, torqueri: licet secus, si indicia contra eundem laborantia essent multum graviora, E urgentiora". ${ }^{14}$

11 Sententiarum l. V, 64, 12, cit. da VERRI, Osservazioni sulla tortura, $2^{a}$ ed., a cura di Barbarisi, Milano 1993, p. 84.

12 E con questo - preciserà il MANZONI nel capit. II della Storia della colonna infame - $\mathrm{i}$ doctores "non intendevan già di proporre un principio, di stabilire una teoria, ma d'enunciar semplicemente un fatto".

13 All'opposto - e come ricorderà MANZONI nel già richiamato capitolo II Farinacio "dava nelle furie" (non possum nisi vehementer excandescere) "contro quei giudici che tengono per lungo tempo legato il reo, prima di sottoporlo alla tortura; e con quella preparazione la rendon più crudele”.

14 Praxis et theoricae criminalis - Partis Primae Tomus Secundus, ed. ultima, Lugduni MDCXIV, quaest. XXXVIII, n. 122. 
Quel che qui preme sottolineare è che l'autore della Cautio criminalis prospetta i predetti criteri di valutazione degli indizi ad torturam, e quindi, in definitiva almeno di riduzione degli spazi di quella pratica, sintetizzando il pensiero di Farinacio col richiamo concettuale unificante - da quest'ultimo non espressamente operato - alla necessità di "sempre privilegiare la presunzione d'innocenza" ${ }^{15}$ Come a dire che il favor che ispira la valutazione di cui si diceva discende pur sempre (come regola minore) da quel principio fondamentale, cui è giusto mettere in collegamento la fama del nostro padre gesuita a titolo di precursore. ${ }^{16}$ Affrontando la questione XXXIX egli ha così modo di precisare: "Dò per presupposto che nessuno possa essere condannato se non risulti con certezza che è colpevole". Ed aggiunge: "neque enim innocens occidi debet, innocens autem omnis esse presumitur, qui nocens esse nescitur".

5. Ma l'impegno del padre Spee contro la tortura - la definisce come "onnipotente" 17 - non si appaga del criterio limitativo della elisione degli indizi, e va ben oltre.

Non per nulla di lui s'è detto che egli rappresenta "forse la più bella e nobile figura fra quante la lunga storia della lotta per l'abolizione della tortura possa vantare". ${ }^{18}$

Intorno a quell'argomento centrale il nostro autore stende e sviluppa, infatti, una fittissima rete di critiche, di repliche e di confutazioni, entro e mediante la quale si alternano e combinano temi e toni di riflessione pacata e di vigore polemico.

A titolo esemplificativo, basterà riportare un piccolo brano tratto dalla questione XX: “... Ci stupiamo che ci siano streghe ovunque?

15 Si veda la p. 112 della traduzione della Cautio criminalis di cui alla nota (2).

16 E' stato anche notato che, con la fondazione della Compagnia di Gesù, "l'abolizione della tortura doveva fare dei grandi passi”: così MELLOR, La torture, Parigi 1949, p. 150, il quale accomuna Spee, in tale ordine di idee, ai padri Tanner e Playman, mentre FIORELLI, op. cit., p. 244, tra i "coraggiosi precursori" fa anche i nomi di Graefe e Keller.

17 E spiega, nell' "Appendice” finale: “... In verità si raccontano in giro numerosi casi di imputati che, sopraffatti dalle torture, hanno confessato di aver commesso crimini assolutamente falsi e che sono stati condannati per l'omicidio di persone che poi si è scoperto essere vive e vegete".

18 FIORELLI, op. cit., p. 232 ss. Nella restrittiva elencazione riguardante gli "uomini illuminati che apertamente mostrarono la disapprovazione loro all'uso della tortura" il VERRI, op. cit., p. 100 ss. non menziona il padre Spee. 
Meravigliamoci piuttosto della straordinaria cecità che regna in Germania e della stupidità degli esperti. Questi ultimi, purtroppo, se ne stanno tranquilli e con tutti i loro agi a scrivere i loro commenti dietro alla stufa senza essere nemmeno sfiorati nell'animo dalla sofferenza e a distribuirla con tanta abbondanza, come farebbe un cieco che parlasse di colori di cui non ha la più pallida immagine". Ed ancora: "Ma perché cerchiamo tanto affannosamente le streghe? Andiamo, giudici, vi dirò io, in quattro e quattr'otto, dove sono.

Prendete i Cappuccini, i Gesuiti, e tutti i religiosi, torturateli, confesseranno. Se alcuni di loro negano, ripetete tre o quattro volte la tortura, confesseranno. Si si dimostrassero ostinati, esorcizzateli, rapateli: si servono di arti magiche, è il diavolo che li rende insensibili. Andate avanti imperterriti, alla fine confesseranno ... Se ancora non vi bastano, ci penserò io a torturare voi, e poi voi torturerete me. Sono sicuro che confesseranno tutti. Così saremmo tutti colpevoli di magia".

A questo punto, proprio tutti - compresi i meno capaci di recepire l'ironia - dovrebbero aver capito.

Sembra poi il caso di limitarci ai titoli delle "questioni" nelle quali l'argomento è più direttamente ripreso e sviluppato: "Se si possa sottoporre più volte a tortura chi è accusata di magia" (quest. XXI); "Perché oggi tanti giudici assolvono con difficoltà le imputate, anche quando si sono purgate attraverso la tortura" (quest. XXII); "Sulla base di quali pretesti si possa ottenere di poter ripetere la tortura in assenza di nuovi indizi (quest. XXIII); "Se il maleficio del silenzio sia un ulteriore indizio valido per la ripetizione della tortura" (quest. XXV); "Se la tortura sia un metodo atto a scoprire la verità" (quest. XXVII); "Se si debba del tutto eliminare la tortura, considerando i pericoli che comporta" (quest. XXIX); "Per quali motivi è consentito procedere alla tortura" (quest. XXXII) ecc. ecc. ${ }^{19}$

19 In particolare, nella trattazione della questione XXXVI l'autore si preoccupa di confutare l'opinione, da tempo abbastanza diffusa fra i giuristi del diritto comune (e fa i nomi di Marsili, Menochio, Monticelli, Mascardi), secondo cui, in caso di delitti particolarmente efferati l'imputato può venir torturato in base a deboli indizi. Al contrario, ed allargando il discorso al tema delle prove, egli scrive: "Che nei delitti efferati, occulti e difficili da dimostrare siano sufficienti prove minori che negli altri casi è talmente lontano dalla verità che anzi, secondo i dettami della retta ragione, sono in questo caso necessarie prove maggiori”. Vi faranno eco, tra gli altri, e pur senza nominarlo, Gaetano Filangieri e Jeremy Bentham, opponendosi ai criminalisti secondo i quali In 
Di contro a tante questioni, ed a così ampio argomentare, prende risalto, per la sua estrema concisione, quanto Montesquieu, nell'Esprit des lois, dirà più tardi (1. VI, capit. XVII): "Contro questa pratica hanno scritto così tante persone provvedute e tante belle menti, che dopo di esse io non oso parlare. Io stavo per dire che essa [la tortura] si adatterebbe ai governi dispotici, ove tutto ciò che ispira terrore maggiormente si insinua entro gli apparati del governo; io stavo per dire che gli schiavi presso i Greci e presso i Romani ...". E seguono sùbito alcuni puntini di sospensione, che peraltro chiudono risolutamente il discorso con un memorabile "basta"!, col dire: "Ma io sento la voce della natura che grida contro di me" (qui crie contre moi).

E non è un caso, poi, che un grande estimatore di Montesquieu, ovvero Cesare Beccaria, abbia affrontato il tema della presunzione d'innocenza proprio in rapporto al tema della tortura. "Un uomo egli scriverà nel $\mathbb{X V I}$ di Dei delitti e delle pene - non può chiamarsi reo prima della sentenza del giudice; né la società può togliergli la pubblica protezione, se non quando sia deciso ch'egli abbia violati i patti coi quali le fu accordata. Quale è dunque quel diritto, se non quello della forza, che dia la podestà ad un giudice - e qui Beccaria riprende l'argomento di Agostino (De civitate Dei, l. XIX, cap. 6) - di dare una pena ad un cittadino, mentre si dubita se sia reo o innocente? (...) o il delitto è certo o incerto: se certo, non gli conviene altra pena che la stabilita dalle leggi, ed inutili sono i tormenti, perché inutile è la confessione del reato; se è incerto, e non devesi tormentare un innocente, perché tale è secondo le leggi un uomo i di cui delitti non sono provati". ${ }^{20}$

atrocissimis leviores conjecturae sufficiunt, et licet judici jura transgredi. Scriveva FILANGIERI, La scienza della legislazione, t. III, Genova 1798, p. 235; vol. I, Milano 1855, p. 501: "Noi abbiamo dunque una resistenza maggiore da superare per commettere un delitto più grave. Tra due accuse dunque l'una di un delitto più atroce, l'altra di un delitto meno atroce, la legge dovrebbe piuttosto ricercare maggiori pruove nella prima che nella seconda". E BENTHAM: Traité des preuves judiciaires, t. II, $3^{a}$ ed., Bruxelles 1840, capit. XV, p. 353: "Più il delitto è grave, per colui al quale lo si addebita, meno è probabile: e c'è allora bisogno che la prova sia più forte”.

20 Ricordiamo anche il VOLTAIRE del Commentario a Beccaria, laddove, trattando, nel $\mathbb{X}$ XII, della tortura giudiziaria, scriveva: "Io ho vergogna di aver parlato sopra questo soggetto dopo quello che ne ha detto l'Autore de' Delitti e delle pene. Io devo restringermi a desiderare, che si rilegga l'Opera di questo amatore dell'umanità". 
Dovranno passare ancora un po' di anni, e finalmente, come si sa, ad abolire, nel continente europeo, la tortura giudiziaria, provvederanno alcuni sovrani illuminati, ${ }^{21}$ - e noi sappiamo da chi illuminati - ai quali la storia della civiltà riserba viva memoria e profonda gratitudine.

21 A tale riguardo v. MASSETTO, La tortura giudiziaria nella dottrina lombarda dei secoli XVI-XVII, ora in Scritti di storia giuridica di Gian Paolo Massetto, t. II, Milano 2018, p. 1132, nota (24). 